\title{
Patente, Gewinnverschiebung und Steuervermeidung
}

\section{Philine Widmer}

\section{Relevanz}

Innovationen treiben die Unternehmensentwicklung. Der größte Teil der F\&E-Aufwendungen wird in multinationalen Unternehmen mit vielen Standorten getätigt. Diese haben steuersparende Möglichkeiten, den F\&E-Aufwand in Ländern mit hoher Steuerbelastung zu tätigen, aber die Patente an Standorte in Niedrigsteuerländern zu übertragen, um dort die Patenterträge gering $\mathrm{zu}$ versteuern. Diese steuermindernde Gewinnverschiebung bringt die Hochsteuerländer unter Druck und schafft Probleme in der steuerlichen Wettbewerbsneutralität zwischen nationalen KMUs und großen Multis. Um steuerliche Innovationsförderung z. B. im Rahmen von Patentboxen mit einem fairen Steuerwettbewerb zu vereinbaren, könnte eine begünstigte Besteuerung von Patenterträgen nur dann erlaubt sein, wenn auch der steuermindernde F\&E-Aufwand im selben Land stattfindet.

Christian Keuschnigg

\section{Quelle}

Der nachfolgende Text ist eine Zusammenfassung von: Karkinsky, Tom und Nadine Riedel (2012), Corporate Taxation and the Location of Patents within Multinational Firms, Journal of International Economics 88, 176-185.

P. Widmer $(\bowtie)$

Universität St. Gallen, St. Gallen, Schweiz

E-Mail: philine.widmer@unisg.ch 
Um sich im Wettbewerb mit einem Qualitätsvorsprung oder Kostenvorteil durchzusetzen, betreiben innovative Unternehmen hohen F\&E-Aufwand. Damit nicht andere Konkurrenten die Ideen nachahmen oder gar stehlen können, müssen sie ihre Erfindungen mit Patenten schützen. In einer innovativen Wirtschaft spielt daher geistiges Eigentum eine immer wichtigere Rolle, vor allem in multinationalen Unternehmen („Multis“), wo sich ein großer Teil der F\&E-Aufwendungen konzentriert. Multis haben viele Standorte weltweit und müssen zur Konzernsteuerung den Unternehmenserfolg zwischen den Tochtergesellschaften klar abgrenzen. Daher müssen die Konzerntöchter, die ein Patent nutzen, eine Lizenzgebühr an jene Einheit des Konzerns zahlen, welche das Patent besitzt. Dabei muss der rechtliche Inhaber des Patents nicht automatische jene Tochtergesellschaft sein, welche den vorherigen F\&E-Aufwand getätigt hat. Die F\&E-Einheit kann das Patentrecht an eine andere Konzerneinheit zur weiteren Verwertung abtreten. Für alle diese Transaktionen sind interne Verrechnungspreise festzusetzen, damit eine Konzernsteuerung mit einer klaren Erfolgsabgrenzung zwischen den Teilbetrieben möglich wird. Da die Transaktionen innerhalb des Konzerns und nicht zwischen unabhängigen Anbietern und Nachfragern stattfinden, gibt es keine „objektiven“"Marktpreise, sondern eben nur Verrechnungspreise.

Unterschiede in der Steuerbelastung haben Einfluss darauf, welchen Tochtergesellschaften der Konzern das Patent zur Verwertung überträgt, und welche Lizenzgebühren bzw. Verrechnungspreise von den anderen nutzenden Einheiten zu zahlen sind. Gewinnstreben und globaler Wettbewerb zwingen die Multis, bei allen Kosten und damit auch bei der weltweiten Steuerbelastung zu „sparen“. Steuerunterschiede schaffen also einen Anreiz, Gewinne von Hochsteuerländern in Niedrigsteuerländer zu verschieben. Multis können erstens die Patente an Tochtergesellschaften in Niedrigsteuerländern zur Verwertung übertragen. Diese erhalten Lizenzzahlungen von allen anderen Konzernstandorten, welche die Patente nutzen. Die Lizenzgebühren steigern die Kosten und reduzieren die Gewinne der Nutzer in den Hochsteuerländern und erhöhen die Einnahmen und den Gewinn der Verwertungsgesellschaft im Niedrigsteuerland. Die gesamte Steuerbelastung sinkt, weil ein größerer Teil des weltweiten Gewinns mit geringen Steuersätzen und ein kleinerer Teil mit hohen Sätzen belastet werden.

Ein zweiter Weg der Gewinnverschiebung ist, die Lizenzgebühr übertrieben hoch anzusetzen. Auch das reduziert den Gewinn der Patentnehmer in den Hochsteuerländern und steigert den Gewinn der Verwertungsgesellschaft im 
Niedrigsteuerland. ${ }^{1}$ Für Steuerbehörden ist es in der Regel schwierig festzustellen, was eine richtige Lizenzgebühr ist, weil sie nur beschränkten Einblick in das Konzerngeschehen haben. Die Nutzungsrechte werden ja innerhalb des Konzerns zu einem internen, selbst kalkulierten Verrechnungspreis zugeteilt und eben gerade nicht auf dem freien Markt zwischen unabhängigen Unternehmen gehandelt, wo sich ein „objektiver Marktpreis“ (sog. Arm's Length Preis) bilden würde. Das Fehlen vergleichbarer Marktpreise gibt den Multis erheblichen Spielraum, mit der Festsetzung von Lizenzgebühren Gewinne von Hoch- in Niedrigsteuerländer zu verschieben und die weltweite Steuerbelastung zu reduzieren.

\begin{abstract}
Multis können Patente an Tochtergesellschaften in Niedrigsteuerländern übertragen, wo die Lizenzerlöse gering besteuert werden. Zusätzlich können sie die Lizenzgebühren festsetzen, um Gewinne von Hochsteuerländern in Standorte mit niedrigen Steuern zu verschieben und die konzernweite Steuerschuld zu senken.
\end{abstract}

Die Forscher finden Evidenz dafür, dass die Multis geistiges Eigentum strategisch ansiedeln. Sie zeigen, dass die Zahl der Patentanmeldungen einer Gesellschaft negativ durch den Steuersatz auf die Patenteinnahmen in ihrem Sitzstaat beeinflusst wird. Der Beitrag dieser Arbeit liegt darin, dass sie den Zusammenhang zwischen Unternehmensbesteuerung und dem Standort von Patentinhabern durch eine umfassende quantitative Studie belegt. Konkret untersucht die Forschungsarbeit einen Datensatz mit 9145 europäische Gesellschaften, die zu multinationalen Unternehmen gehören, im Zeitraum zwischen 1995 und 2003. Es ergeben sich über 60.000 Beobachtungen. Berücksichtigt werden Gesellschaften, welche über den Zeitraum mindestens einmal ein Patent angemeldet. Die geografische Verteilung der Patentinhaber in Europa widerspiegelt die Größe der Länder. Die meisten befinden sich in Frankreich, Deutschland und Großbritannien, aber auch die Schweiz und die Niederlande weisen eine hohe Anzahl auf.

Die Analyse untersucht den Zusammenhang zwischen drei Größen, nämlich Informationen über die Beteiligungsverhältnisse der Gesellschaften, Anmeldungen beim Europäischen Patentamt und Unternehmenssteuersätze im Land

\footnotetext{
${ }^{1}$ Sollte das Patent im Besitz einer Tochter in einem Hochsteuerland sein, dann kann die Lizenzgebühr besonders niedrig angesetzt werden, damit dort möglichst geringe Einnahmen und wenig Gewinn entstehen. Dafür bleiben die Lizenzkosten der anderen Töchtern in Ländern mit niedriger Steuerbelastung gering und die Gewinne dort hoch. Wiederum wird Gewinn von Hoch- zu Niedrigsteuerländern verschoben.
} 
der Niederlassung. Die Forscher qualifizieren Tochtergesellschaften als solche, sobald sie zu mindestens $25 \%$ von einer anderen Gesellschaft kontrolliert werden. Die oberste Gesellschaft in einer solchen Eigentümerkette wird als Muttergesellschaft identifiziert. Sie dokumentieren, wie viele Patente eine Gesellschaft pro Jahr beim Europäischen Patentamt anmeldet. Im Durchschnitt sind es 0,9 Patente. Schließlich erfassen sie die absolute und relative steuerliche Attraktivität eines Landes. Der durchschnittliche Steuersatz beträgt $39 \%$ und variiert zwischen 10 und $59 \%$. Neben dem Steuersatz auf Patenteinnahmen im jeweiligen Land wird auch das Differential der Steuersätze zu anderen Ländern berechnet. Konkret ermitteln sie die durchschnittliche Differenz zwischen dem Steuersatz für eine Gesellschaft und den Steuersätzen aller anderen Tochtergesellschaften desselben Multis in anderen Standortländern.

Die Forscher schätzen, dass ein höherer Steuersatz auf Patenteinnahmen die Zahl der Patentanmeldungen an diesem Standort signifikant verringert. Die Erhöhung des Steuersatzes um zehn Prozentpunkte reduziert die Zahl der Anmeldungen um $35 \%$. Auch ein höheres Steuerdifferential hat einen signifikant negativen Einfluss. Die Patentanmeldungen gehen also auch dann stark zurück, wenn ein Land zwar seinen eigenen Steuersatz unverändert lässt, aber andere Standortländer ihre Steuersätze senkt. Auch damit steigt der Anreiz, die Patente in den Ländern mit tieferer Steuerbelastung anzumelden, weil dort die Patenterträge günstiger besteuert werden. Um robuste Ergebnisse zu erhalten und andere Einflüsse auf die Patentansiedlung herauszufiltern, schätzen die Autoren eine Reihe von unterschiedlichen Spezifikationen und kontrollieren für Einflüsse von über die Zeit konstanten Charakteristika einer Gesellschaft sowie für jahresspezifische Einflüsse.

Eine Erhöhung des Steuersatzes auf Patenteinnahmen um zehn Prozentpunkte verringert die Anzahl an Patentanmeldungen um $35 \%$.

Insbesondere die Hochsteuerländer versuchen, die Aushöhlung ihrer Steuerbasis durch Gewinnverschiebung zu verhindern. Die Analyse berücksichtigt auch rechtliche Regelungen wie z. B. Quellenbesteuerung von Patenteinnahmen, welche die effektive Steuerbelastung und damit die Standortentscheidung für Patente beeinflussen. Wenn Lizenzgebühren grenzüberschreitend bezahlt werden, kann der Sitzstaat des Lizenznehmers eine Quellensteuer auf die gezahlten Gebühren erheben. Um eine Doppelbesteuerung zu vermeiden, gewährt der Sitzstaat des Patentinhabers in solchen Fällen meist eine Steueranrechnung und behandelt die Quellensteuer im anderen Land als Steuervorauszahlung. Daher bestehen Anreize, Patente an Standorten mit bilateralen Steuerabkommen, welche eine 
großzügige Anrechnung der bereits in anderen Ländern bezahlten Quellensteuern gewähren, anzusiedeln. Die Forscher berechnen für jede Gesellschaft den Steuersatz, der zur Anwendung käme, wenn sie eine Lizenzzahlung von jeder anderen Tochtergesellschaft erhalten würde. Die zusätzlichen Schätzungen bestätigen den negativen Einfluss höherer Steuern auf die Patentanmeldungen.

Es kommt nicht nur auf die heimische Steuerbelastung an. Ein Land verliert auch dann Patente, wenn das Ausland die effektive Steuerbelastung auf Patenterträge senkt.

Um ihr Steueraufkommen zu schützen, versuchen Hochsteuerländer, eine Gewinnverschiebung auch mit einer Hinzurechnungsbesteuerung (sog. Controlled Foreign Company Regeln, CFC) einzudämmen. Damit können unter bestimmten Bedingungen Einnahmen von ausländischen Konzerntöchtern am Standort der Muttergesellschaft nachversteuert werden. Eine solche Nachversteuerung erfolgt, wenn eine Tochter in einem Niedrigsteuerland weniger als $60 \%$ der Steuer zahlt, die im Sitzstaat der Muttergesellschaft anfallen würde. Jedoch ist diese Regel nur für 2,2 \% der Beobachtungen im Datensatz bindend. Die meisten Konzerntöchter sind eben aus anderen wirtschaftlichen Gründen in europäischen Staaten angesiedelt, wo die Steuern ohnehin tendenziell hoch sind. Die entsprechende Anpassung erhöht den Steuersatz nur marginal und bestätigt die empirischen Resultate sowohl quantitativ als auch qualitativ.

Die Forscher präsentieren robuste empirische Ergebnisse dafür, dass Patente verstärkt in Staaten mit einer niedrigen Steuerbelastung der konzerninternen Lizenzeinnahmen angemeldet werden. Dies lässt die vermutete Gewinnverschiebung plausibel erscheinen. Die strategische Ansiedelung von Patenten multinationaler Unternehmen schafft Anreize für Staaten, durch attraktive Steuersätze um Gesellschaften mit Patenten zu konkurrieren. Großbritannien zum Beispiel bietet seit 2013 eine sogenannte Patentbox an, welche es Firmen erlaubt, Gewinne aus Patenten zu einem ermäßigten Steuersatz zu versteuern. Auch in der Schweiz sollen mit der geplanten Unternehmenssteuerreform III Erträge aus Patenten privilegiert behandelt werden. Staaten mit hohen Steuern und vielen F\&E-intensiven Unternehmen könnten unter Druck kommen, die Abwanderung von Patenten und anderen immateriellen Gütern zu verhindern. Eine Möglichkeit dafür ist die Nachversteuerung von Patenterträgen nach den oben beschriebenen CFC-Regeln, die unter anderem in den USA und in Deutschland eingeführt wurden. Auch die Bemühungen im Rahmen der OECD zielen darauf ab, missbräuchliche Steuervermeidung von Multis und besonders aggressive Formen des Steuerwettbewerbs der Staaten einzudämmen. Es bestehen Bedenken, dass Patentboxen vorwiegend 
Anreize zur Gewinnverschiebung setzen und die Staaten sich gegenseitig Patenterträge zur Stärkung der eigenen Steuerbasis abjagen, ohne die Forschung und Entwicklung wirklich zu fördern. Man kann den Steuerwettbewerb als unfair betrachten, wenn ein Staat Steuerausfälle aus dem Abzug von F\&E-Abwendungen hinnehmen muss und andere Staaten nur die Patenterträge besteuern, weil die Multis ihre Patente dorthin verschieben. Unter anderem wird deshalb gefordert, dass Patentboxen zwecks Innovationsförderung nur mit der Einschränkung erlaubt sein sollen, dass Patenterträge und der dazu notwendige F\&E-Aufwand im selben Land stattfinden.

Open Access Dieses Kapitel wird unter der Creative Commons Namensnennung 4.0 International Lizenz (http://creativecommons.org/licenses/by/4.0/deed.de) veröffentlicht, welche die Nutzung, Vervielfältigung, Bearbeitung, Verbreitung und Wiedergabe in jeglichem Medium und Format erlaubt, sofern Sie den/die ursprünglichen Autor(en) und die Quelle ordnungsgemäß nennen, einen Link zur Creative Commons Lizenz beifügen und angeben, ob Änderungen vorgenommen wurden.

Die in diesem Kapitel enthaltenen Bilder und sonstiges Drittmaterial unterliegen ebenfalls der genannten Creative Commons Lizenz, sofern sich aus der Abbildungslegende nichts anderes ergibt. Sofern das betreffende Material nicht unter der genannten Creative Commons Lizenz steht und die betreffende Handlung nicht nach gesetzlichen Vorschriften erlaubt ist, ist für die oben aufgeführten Weiterverwendungen des Materials die Einwilligung des jeweiligen Rechteinhabers einzuholen.

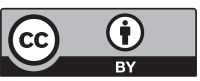

